\title{
Article
}

\section{Intraoperative Adjuvant Hyperthermic Intraperitoneal Chemotherapy in Patients with Locally Advanced Colon Cancer: A Prospective Parallel Phase II Study.}

Ina Macaione 1,2, Lucio Mandalà 2, Antonella Campisi ${ }^{3}$, Elena Roz ${ }^{4}$, Sebastiano Mercadante 5, Dario Piazza 6 , Pietro Mezzatesta ${ }^{2}$ and Vittorio Gebbia ${ }^{7,8^{*}}$

1. Department of Surgical, Oncological and Stomatological Disciplines, University of Palermo, Palermo, Italy.

Department of Surgery, La Maddalena Cancer Center, Palermo, Italy inamacaione@gmail.com

2. Department of Surgery, La Maddalena Cancer Center, Palermo, Italy lmandala@yahoo.it, pmezzatesta@virgilio.it

3. Department of Radiology, La Maddalena Cancer Center, Palermo, Italy, antocampisi@yahoo.it

4. Department of Pathological Anatomy and Oncological Molecular Biology, La Maddalena Cancer Center, Palermo, Italy elena roz@hotmail.com

5. Anesthesia and Intensive Care Unit, Pain Relief and Palliative Care Unit, La Maddalena Cancer Center, Palermo, Italy terapiadeldolore@lamaddalenanet.it

6. GSTU Foundation for Cancer Research, Piazza Sturzo n. 15, 90100 Palermo, Italy, dariopiazza@gmail.com

7. Department of Oncology, La Maddalena Cancer Center, Palermo, Italy vittorio.gebbia@gmail.com

8. Oncology Section, Department Promise, University of Palermo, via del Vespro n. 15, 90100 Palermo, Italy

(*) Corresponding author: Prof. Vittorio Gebbia, Medical Oncology and Supportive Care Unit, La Maddalena Cancer Center and PROMISE Department - University of Palermo, Palermo, Italy. Via S. Lorenzo, 312/D, 90146 Palermo PA Italy; Tel.: 0039330696205

E-mail: vittorio.gebbia@gmail.com

\begin{abstract}
.
Background: Prophylactic hyperthermic intraperitoneal chemotherapy (HIPEC) showed promising results in patients with colorectal carcinoma at high risk of recurrence but still without clinically and radiologically evident signs of peritoneal spread. This study aims to analyze the feasibility of this proactive, early phase, multimodality approach.

Methods: A mono-institutional, prospective, parallel, two-stage phase II trial enrolled 49 patients to standard surgery or surgery plus intraoperative HIPEC. Before the procedure and during surgery, patients received intravenous fluorouracil (and leucovorin to potentiate oxaliplatin activity. Data analysis included length of hospital stay, surgery duration, type of surgery and chemotherapy-related complications risk score.

Results: No significant difference was seen in the median time spent in the hospital with a median stay of 7 days in both groups ( $\mathrm{p}=0.5720)$. The surgical procedure's median duration was longer in the HIPEC group than in the control one. Side-effects and surgical complications did not cross at any time the Pocock-type boundary for side/effect monitoring ( $\mathrm{p}=0.80$, N.S.). Conclusions: The present prospective study results demonstrate the feasibility and safety of the colorectal surgery plus HIPEC treatment in patients with colorectal cancer patients at high-risk for peritoneal invasion, although clinically and radiologically undetectable.
\end{abstract}

Keywords. Colorectal cancer, peritoneal metastasis, HIPEC, surgery, feasibility. 


\section{Introduction}

Globally, colorectal cancer (CRC) is the third most common cancer in males and the second in females, with 1.8 million new cases and almost 861,000 deaths in 2018 (1). Its incidence and mortality rates vary markedly around the world (1). The usual recurrence sites include anastomotic site, mesentery or nodal basin, retroperitoneum, and peritoneum (2). Peritoneal carcinomatosis (P.C.) may reach up to $25-30 \%$ of all recurrences after potentially curative resection (3) and carries a dismal prognosis with a survival of 5 months if untreated and has a range between 5 and 15 months if treated with palliative systemic therapy (4). The peritoneal spread is an event that involves $10-15 \%$ of CRC patients at the time of surgery. Occult PC due to the spread of metastatic cells from full-thickness bowel wall invasion or "in transit" from lymphatic vessels during surgical dissection is the main problem. Several features of the primary colorectal tumor represent risk factors in developing PC. These features include mucinous or signet ring cell carcinoma, locally advanced disease (T4 stage), tumor perforation, nodal stage, right-sided tumor location, and non-radical resection (R1 or R2) (5-9). At present, surgery followed by hyperthermic intraperitoneal chemotherapy (HIPEC) represents one of the best therapeutic options for patients with PC. The rationale of this multimodality strategy is to remove all visible peritoneal tumor deposits surgically and to eradicate microscopic residual disease with HIPEC (10). The effectiveness of HIPEC depends on the grade of completeness of cytoreduction and the extension of peritoneal disease spread, which is quantified by the Peritoneal Cancer Index (PCI) scoring system. PCI is a diagnostic and prognostic system that is a sum of scores in thirteen abdominal regions. Each receives a score of 0-3 based on the largest tumor size in each area, with a range from 0 to 39 . Higher scores indicate more widespread and larger tumors in the peritoneal cavity. A large study by Klaver et al. showed that patients with low PCI (0-5) might have lower postoperative morbidity and a 5-year survival rate of 70\%. In comparison, those of the patients with high PCI $(>16)$ reach only a 10\% 5-year survival rate with a higher rate of complications (11). Although this study showed no survival advantage of postoperative HIPEC, prophylactic HIPEC highlighted promising results in patients at high risk of recurrence but still without clinically and radiologically evident signs of peritoneal spread and encouraged the encouraging results as the theoretical benefit of in these patients (12-15).

In this paper, we report the results of a study of the feasibility and safety of colorectal surgery plus simultaneous intraoperative adjuvant hyperthermic intraperitoneal chemotherapy in patients at high risk of recurrence but still without evident signs of peritoneal spread in terms of length of hospital stay, surgical and medical treatment-related toxicity.

\section{Patients and methods.}

\subsection{Study design.}

This study is a mono-institutional, prospective, parallel, two-stage phase II trial aimed to evaluate the safety and efficacy of adjuvant intraoperative adjuvant hyperthermic intraperitoneal chemotherapy in patients with locally advanced colon cancer who were at high-risk for peritoneal carcinomatosis and underwent cytoreductive surgery. After the approval by the local Ethics Committee of Palermo University $\left(\mathrm{n}^{\circ} 10 / 18-\right.$ 14/11/2018), this study was carried out according to the Declaration of Helsinki. All participants included in the study signed a specific, informed consent.

Patients with colon cancer or intraperitoneal rectosigmoid cancer $(15 \mathrm{~cm}$ from the anal verge) with clinical T3/T4 N0-2 M0 stage or perforated colon cancer were prospectively enrolled between January 2019 and October 2020. Eligibility criteria also included: age between 18 and 75 years; ECOG performance status of 0-2, adequate renal, hepatic and bone marrow function, no major uncontrolled metabolic, cardiovascular or neurologic diseases, histologically proven adenocarcinoma, cancer with mucinous or signet ring cell components, minimal synchronous P.C. - nodules $<1 \mathrm{~cm}$ in the omentum and close to the primary tumor synchronous ovarian metastases. PC and ovarian tumor deposits must have been macroscopically wholly resected at the same time as the primary tumor. Exclusion criteria were a distant metastatic disease, extensive P.C., second malignancies, and-active infection or severe associated medical conditions. 


\subsection{Clinical outcomes evaluation.}

The study endpoints of the first-stage were length of hospital stay, surgical and medical treatmentrelated toxicity after adjuvant HIPEC.

Safety was reported according to the Dindo et al. classification for surgically related complications and to the National Cancer Institute Common Toxicity Criteria (NCI-CTC) version 4.0 for chemotherapy-related adverse events $(16,17)$. The surgical complication observation period included 30 days after the surgical procedure. Surgical complications and adverse events occurring within 30 postoperative days or during the same hospital stay were graded from zero to five. Follow-up assessments took place every three months with clinical evaluation, thoracic/abdominal C.T. scan and markers measurements, endoscopic procedures, or as needed accordingly to oncologists' evaluation.

\subsection{Treatments.}

Potentially eligible patients were identified through an intensive preoperatory workup, including clinical history, colonoscopy, thoracic, abdominal, pelvic C.T. scan, and serum markers (CEA; Ca19.9). Colon resection was done according to the oncological principles of adequate lymphadenectomy; tumor deposits on visceral and parietal surfaces were surgically removed and organ resections as surgically needed. Intraoperative pathologic evaluation assessed tumor depth, and the histologic feature was mandatory to include patients in the study. At the end of the surgery, patients who had signed informed consent and acceptance of receiving intraperitoneal chemotherapy received HIPEC.

HIPEC was delivered with the closed technique with oxaliplatin at the dose of $460 \mathrm{mg} / \mathrm{m} 2 \mathrm{in} 21 / \mathrm{m} 2$ of dextrose solution over 30 minutes at a flow rate of $2 \mathrm{~L} / \mathrm{min}$ and a temperature of $43^{\circ} \mathrm{C}$. Before the HIPEC procedure and during surgery, patients received intravenous fluorouracil of $400 \mathrm{mg} / \mathrm{m} 2$ and leucovorin of 20 $\mathrm{mg} / \mathrm{m} 2$ to potentiate oxaliplatin activity. Systemic adjuvant chemotherapy with FOLFOX or XELOX regimens was also given to patients treated with HIPEC and control ones if indicated according to treatment guidelines.

\subsection{Statistics.}

The size of this study (two independent samples) was calculated employing a probability of a type-1 error $10 \%$ cutoff (alpha 0.1 ), and a probability of a type-2 error related to the study power (power $1-\beta$ ) cutoff of $20 \%$ cutoff (beta 0.80 ) (18). The endpoints were binomial, therefore for an anticipated mean of $3.5 \pm 1.5$ in the control group and an assumed 35\% increase in complication rate in the experimental arm with an enrollment ratio of 2:1, a total of 49 evaluable patients had to be enrolled, at least 14 and 38 in the experimental group and the control one respectively.

Continuous monitoring for toxicity using a Pocock-type boundary was employed with an event probability $(\theta)$ of 0.2 and the desired probability of early stopping of 0.05 (19). The trial will be stopped if the number of dose-limiting toxicities is equal to or exceeds $b_{n}$ out of $n$ patients with completed follow-up. This boundary is equivalent to testing the null hypothesis; after each patient, the event rate is equal to 0.1 , using a one-sided level 0.026846 test. Sequential boundaries were used to monitor the dose-limiting toxicity rate. The accrual will be halted if excessive numbers of dose-limiting toxicities were seen: if the number of dose-limiting toxicities is equal to or exceeds $b_{n}$ out of $n$ patients with full follow-up. As shown in Figure n. 1, this is a Pococktype stopping boundary that yields the probability of crossing the boundary at most [probability of early stopping] when the dose-limiting toxicity rate is equal to the acceptable rate [event probability $\theta$ ].

\begin{tabular}{|l|l|l|l|l|l|l|l|l|l|l|l|l|l|l|l|}
\hline Number of patients & $n$ & 1 & 2 & 3 & 4 & 5 & 6 & 7 & 8 & 9 & 10 & 11 & 12 & 13 & 14 \\
\hline Boundary & $b_{n}$ & - & - & 3 & 4 & 4 & 4 & 5 & 5 & 5 & 6 & 6 & 6 & 7 & 7 \\
\hline
\end{tabular}

Figure n. 1. Pocock-type boundary for side/effect monitoring. The trial will be stopped if the number of severe complications and toxicity is equal or exceed $b_{n}$ out of $n$ patients with complete follow-up. 
A descriptive analysis of all included patients was performed. Patient characteristics, tumor characteristics, and operative findings by lymphadenectomy or other surgical maneuvers were compared using Wilcoxon's test for quantitative variables and chi-square or Fisher's exact tests for qualitative variables. The time-dependent comparison was constructed using the Kaplan-Meier method with the log-rank test and the Gehan-Breslow-Wilcoxon test to detect differences between groups.

\section{Results.}

\subsection{Patient population.}

Overall, 49 patients were enrolled in the study. Table 1 reports the main clinical, demographic, and clinical characteristics of enrolled patients. Eighteen patients were candidate to receive post-surgery HIPEC, and 31 patients had surgery only. Fifteen out of the eighteen patients who were candidates for HIPEC were evaluable $(83 \%)$. Table 1 reports no statistically significant differences between the two groups of patients in terms of gender, median age, the primary tumor site, percentage of patients treated with neoadjuvant chemotherapy or radiotherapy. Nearly one-third of patients had the primary tumor in the ascending colon/hepatic flexure, while the remaining had to descending colon/splenic flexure or sigmoid colon. Three patients had neoadjuvant chemotherapy and only one pre-operative radiotherapy. Video-laparoscopic surgery was done in 33\% and 39\% of HIPEC and control groups, respectively. In some patients it was necessary to combine the resection of other organs with the standard colectomy. Peritoneal washing was cytologically negative in all cases.

Table 1. Patients' demographic and clinical characteristics

\begin{tabular}{|c|c|c|c|c|}
\hline & & HIPEC & CONTROLS & \\
\hline N. of patients & & $18(100 \%)$ & $31(100 \%)$ & \\
\hline N. evaluable patients & & $15(83 \%)$ & $31(100 \%)$ & \\
\hline Age (years) & Median (range) & $58(46-76)$ & $60(44-78)$ & \\
\hline \multirow[t]{2}{*}{ Gender } & Male & $5(36 \%)$ & $17(55 \%)$ & \multirow[t]{2}{*}{$\mathrm{p}=0.275787$} \\
\hline & Female & $9(64 \%)$ & $14(45 \%)$ & \\
\hline \multirow[t]{4}{*}{ Site of primary } & ascending colon* & $6(33 \%)$ & $8(26 \%)$ & \multirow[t]{4}{*}{$\mathrm{p}=\mathrm{NS}$} \\
\hline & transverse colon & 0 & $1(03 \%)$ & \\
\hline & descending colon $* *$ & $3(17 \%)$ & $10(32 \%)$ & \\
\hline & sigmoid/rectal colon & $9(50 \%)$ & $12(39 \%)$ & \\
\hline CEA at basal > 4 & & $2(11 \%)$ & & \\
\hline \multirow[t]{5}{*}{ Neoadjuvant CT } & Yes & $1(6 \%)$ & $1(3 \%)$ & \\
\hline & FOLFOX & 1 & 0 & \\
\hline & XELOX & 1 & 0 & \\
\hline & Capecitabine & 0 & 1 & \\
\hline & None & 13 & 30 & \\
\hline Neoadjuvant R.T. & & 0 & 1 & \\
\hline \multirow[t]{5}{*}{ Surgery } & Open & $12(67 \%)$ & $19(61 \%)$ & \multirow{2}{*}{$\begin{array}{l}\mathrm{p}=0.059126 \\
\mathrm{NS}\end{array}$} \\
\hline & VLS & $6(33 \%)$ & $12(39 \%)$ & \\
\hline & hemicolectomy & 10 & 17 & \multirow[t]{3}{*}{$\mathrm{p}=\mathrm{NS}$} \\
\hline & $\begin{array}{l}\text { rectal anterior } \\
\text { resection }\end{array}$ & 8 & 14 & \\
\hline & Ileostomy & 6 & 5 & \\
\hline \multirow[t]{6}{*}{ Other organs resected } & Pancreas & 1 & 0 & \\
\hline & gall bladder & 1 & 0 & \\
\hline & uterus/ovary & 3 & 3 & \\
\hline & urinary bladder & 2 & 2 & \\
\hline & Peritoneum & 1 & 3 & \\
\hline & Prostate & 1 & 0 & \\
\hline Peritoneal washing & Negative & 14 & 31 & \\
\hline
\end{tabular}




\begin{tabular}{|c|c|c|c|}
\hline & Positive & 0 & 0 \\
\hline Histology & Adenocarcinoma & $18(100 \%)$ & $31(100 \%)$ \\
\hline \multirow[t]{15}{*}{ Post-surgical stage } & pT3, N0, M0 & 5 & 2 \\
\hline & pT3, N1a, M0 & 1 & 6 \\
\hline & pT3, N1b, M0 & 1 & 11 \\
\hline & pT3, N2a, M0 & 2 & 3 \\
\hline & pT3, N2b, M0 & 2 & 3 \\
\hline & pT3, N1c, M0 & 0 & 1 \\
\hline & pT3, N2b, M0 & 0 & 1 \\
\hline & pT3, N0,M1 (per) & 1 & 0 \\
\hline & pT4, N0, M0 & 0 & 1 \\
\hline & pT4a, N1b, M0 & 0 & 2 \\
\hline & pT4b, N0, M0 & 1 & 0 \\
\hline & pT4b, N1a, M0 & 0 & 1 \\
\hline & pT4, N2a, M0 & 1 & 0 \\
\hline & pT4b, N1b, M0 & 1 & 1 \\
\hline & pT4, N2b, M0 & 1 & 0 \\
\hline
\end{tabular}

\subsection{Outcomes.}

Table 2 shows results in terms of duration of surgical procedure subtracting time dedicated to hyperthermic intraperitoneal chemotherapy procedure, length of hospitalization, surgical complications, sideeffects related to chemotherapy, and impact of HIPEC on post-surgical chemotherapy. No significant difference was seen in median time spent in the hospital (Figure 2) with a median stay of 7 days in both groups (Gehan-Breslow-Wilcoxon test $\mathrm{p}=0.5720$, N.S.; Mantel-Haenszel HR 0,0922, 9\%\% CI 0.4282-2.299). As shown in Figure n. 3, the surgical procedure's median duration was longer in the HIPEC group than in the control one (median 192 versus 138 minutes). This difference was statistically significant if the log-rank test applied $(\mathrm{p}=0.0037)$, but not if the Gehan-Breslow-Wilcoxon test used $(\mathrm{p}=0,0810)$. The rate of surgical complications as well side-effects potentially related to chemotherapy were equally distributed in both groups. Side-effects and surgical complications did not cross at any time the Pocock-type boundary for side/effect monitoring ( $\mathrm{p}=0.80$, N.S.). Moreover, HIPEC did not affect subsequent adjuvant chemotherapy's safety being toxicity the same in both groups of patients.

Table 2. Results

\begin{tabular}{|c|c|c|c|c|}
\hline & & HIPEC & CONTROLS & \\
\hline Duration of surgery (minutes) & median (range) & $\begin{array}{c}192 \\
(71-276)\end{array}$ & $\begin{array}{c}148 \\
(77-221)\end{array}$ & $\mathrm{p}=0.0037$ \\
\hline \multirow[t]{2}{*}{ Post-procedure side-effects $^{\circ}$} & Nausea/vomiting & 1 & 2 & \multirow[t]{2}{*}{$\mathrm{p}=\mathrm{NS}$} \\
\hline & None & 14 & 29 & \\
\hline Hospital stay (days) & median (range) & $7(6-21)$ & $7(5-15)$ & $\mathrm{p}=\mathrm{NS}$ \\
\hline Complications after discharge & & $1 \S$ & $1^{\wedge}$ & \\
\hline \multirow[t]{5}{*}{ Adjuvant chemotherapy } & & $11(73 \%)$ & $25(81 \%)$ & \multirow[t]{5}{*}{$\mathrm{p}=\mathrm{NS}$} \\
\hline & FOLFOX & 2 & 4 & \\
\hline & XELOX & 7 & 18 & \\
\hline & CAPECITABINE & 2 & 3 & \\
\hline & none & $4(27 \%)$ & $6(19 \%)$ & \\
\hline Percent of planned cycles & & $90 \%)$ & $87(\%)$ & $\mathrm{p}=\mathrm{NS}$ \\
\hline \multirow[t]{5}{*}{ Side-effects $(\geq$ grade 3 ) } & Mucositis & $2(18 \%)$ & $4(16 \%)$ & \multirow[t]{5}{*}{$\mathrm{p}=\mathrm{NS}$} \\
\hline & Diarrhea & $2(18 \%)$ & $3(12 \%)$ & \\
\hline & Neutropenia & $2(18 \%)$ & $6(24 \%)$ & \\
\hline & Febrile neutropenia & 0 & 0 & \\
\hline & Platelets & 0 & 1 & \\
\hline Delays in chemotherapy & yes & $4(27 \%)$ & $7(28 \%)$ & $\mathrm{p}=\mathrm{NS}$ \\
\hline
\end{tabular}




\begin{tabular}{|l|l|c|c|c|}
\hline & no & & & \\
\hline Adjuvant R.T. & & 1 & 1 & \\
\hline
\end{tabular}

${ }^{\circ}$ chemotherapy-related; $\S$ splenic artery bleeding; ${ }^{\wedge}$ dehiscence of the anastomosis

\section{Lenght of hospital stay}

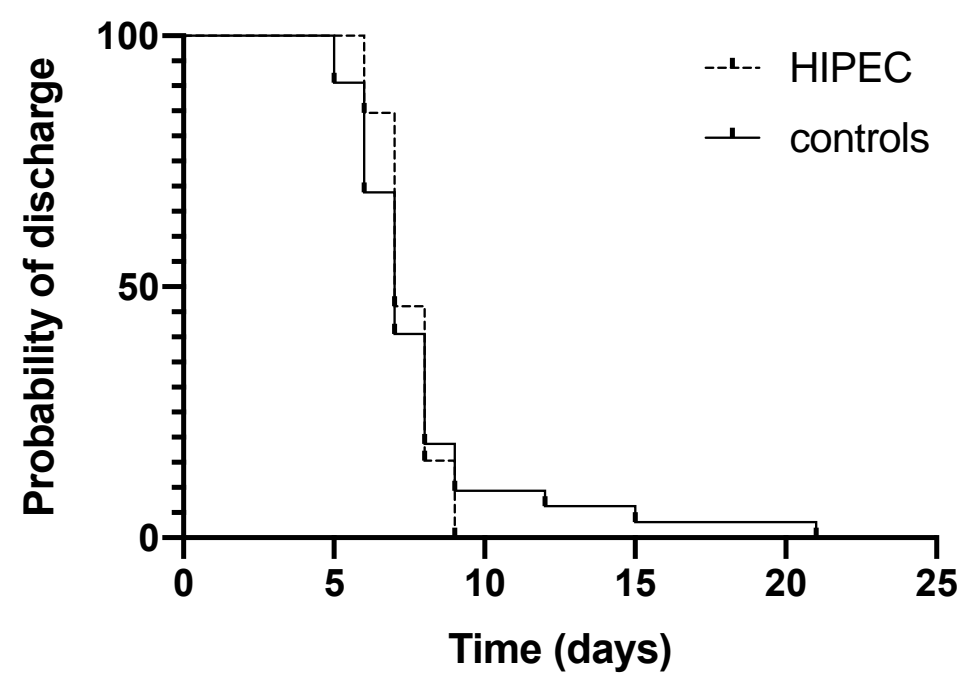

Figure 2.

\section{Duration of surgical procedure}

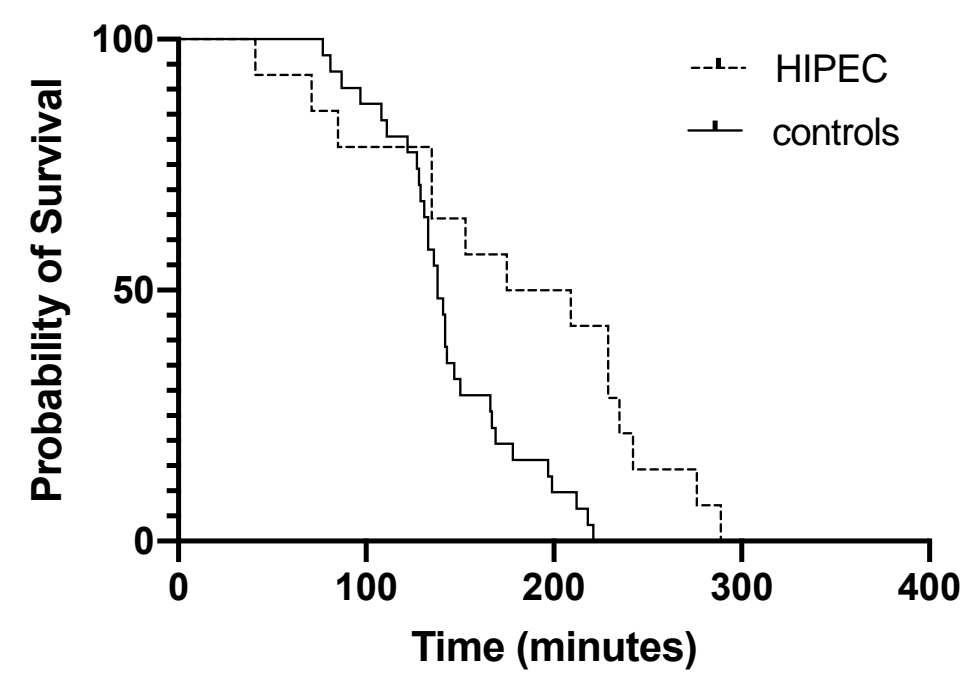

Figure 3. Length of surgical procedure was calculated subtracting time lost to set up and to perform HIPEC.

\section{Discussion.}

Metastatic diffusion into the peritoneal surface carries out a very unfavorable prognosis and a dismal quality of life in many patients with recurrent/metastatic CRC (20). The recent interest in adjuvant HIPEC for CRC at high risk of peritoneal diffusion is a revival of a treatment strategy originating from the 1980s (21). Despite the logical scientific background of adjuvant HIPEC, currently available shreds of evidence are somewhat conflicting. In practice, several factors influence the optimization of this multimodality approach, such as patient characteristics, drug pharmacodynamics, and methodological issues, explaining the outcome differences in published trials (21). Clinical data suggest that one-fifth of patients with locally advanced (T4 stage) or perforated colon cancer are at high risk of developing peritoneal metastases and suitable for surgery 
plus HIPEC with a reasonable chance of improving their outcome $(22,23)$. In cases with detectable PC, the surgical procedure involving peritonectomy and visceral resections must be accurate and result in a complete cytoreduction (21). The majority of studies employed a combination of leucovorin-potentiated fluoropyrimidine and oxaliplatin. This choice relies on well-documented drug synergism, the pharmacokinetic analysis of intraperitoneally delivered oxaliplatin, and the well-documented activity of such regimen (FOLFOX) in the treatment of CRC patients in adjuvant and metastatic settings (24-26).

Researchers at the National Cancer Centre in Singapore have recently published an updated review of the state of the art of surgical management and HIPEC for locally advanced CRC (27). The multicenter COLOPEC study was carried out in nine hospitals in the Netherlands and the primary endpoint was peritoneal metastasis free-survival at 18 months, according to an intention-to-treat (11). This study followed a pilot study, which reported a clear advantage for adjuvant delayed laparoscopic HIPEC after a median followup of 4.5 years in patients with a high risk of peritoneal spread (28). In the COLOPEC trial, 204 patients with clinical or pathological T4, N0-2, M0 stage or perforated CRC were randomized before surgery in a 1:1 fashion to receive adjuvant systemic chemotherapy alone or HIPEC within 5-8 weeks after primary resection followed by standard adjuvant systemic chemotherapy (11). Patients were stratified for perforation, stage of the disease, age (<65 years or $\geq 65$ years), and surgical approach (open or laparoscopic). Adjuvant HIPEC consisted of systemic leucovorin potentiated fluorouracil followed by intraperitoneal hyperthermic delivery of oxaliplatin by either a laparoscopic or open procedure to allow exploration of the abdominal cavity for peritoneal staging and adhesiolysis when necessary. This technical approach was based on the assumption that second-look surgery, first described more than five decades ago, remains the only method to ascertain the presence of minimal progression at the peritoneal surface (28). This study showed no statistically significant difference being peritoneal metastasis-free survival at 18-months, $80.9 \%$ for the HIPEC group versus $76.2 \%$ for the control group, respectively. This study's main criticism is the high proportion (91\%) of patients who received delayed adjuvant HIPEC 5 to 8 weeks after primary tumor resection when adhesions and tumor cell entrapment may have limited the drug distribution and effectiveness of HIPEC. Also, $9 \%$ of the 100 patients in the HIPEC group had peritoneal invasion before delivering HIPEC.

Another recently published prospective open-label, phase III trial (PROPHYLOCHIP-PRODIGE), carried out in France, failed to improve disease-free survival compared to standard surveillance (29). This trial enrolled 150 patients affected by CRC who underwent resection of the primary tumor and synchronous peritoneal or ovarian metastases and treated with adjuvant systemic chemotherapy. Patients were randomized to no further therapy or second-look surgery at the end of chemotherapy, plus, if no recurrence, oxaliplatinor mitomycin-based HIPEC. The study did not reach the primary endpoint being 3-year disease-free survival $53 \%$ (95\% CI 41-64) in the surveillance group versus 44\% (33-56) in the second-look surgery group (hazard ratio $0 \cdot 97,95 \%$ CI $0 \cdot 61-1 \cdot 56)$. These results disfavored the use of second-look surgery plus HIPEC in this clinical setting.

Overall, the results of these two-phase III trials challenged the effectiveness of HIPEC protocol with oxaliplatin and raised the question of whether delayed and limited exposure to chemotherapy may negatively affect its antitumor effect. Timing of adjuvant HIPEC is another point of debate since it should be ideally delivered during primary surgical resection to avoid tumor cell entrapment and delayed microscopic disease management. Statistical sample underpowering could be another explanation for the failure to reach study endpoints.

Although surgery plus HIPEC yielded unsatisfactory results in patients with a high PCI score, it showed promising results in patients at high risk of recurrence but still without clinically evident peritoneal spread. Generally, CRC patients with a low PCI score show better survival rates and lower postoperative morbidity after treatment with surgery and HIPEC (20). Therefore, the recognition and management of peritoneal invasion as early as possible may play a pivotal role in maximizing therapeutic results and, ultimately, patients' survival and quality of life (11). Unluckily early detection of peritoneal invasion is a significant challenge due to the lack of signs and symptoms and the relatively low accuracy of imaging techniques. C.T. scan may detect only less than $30 \%$ of peritoneal deposits with a size $<5 \mathrm{~mm}(21,22)$.

In this paper, we report the feasibility and safety of colorectal surgery plus HIPEC in terms of length of hospital stay, surgical, and medical treatment-related toxicity in a mono-institutional series of CRC patients high risk of recurrence but still without evident signs of peritoneal spread who underwent radical surgery. In 
our hands, colorectal surgery plus HIPEC was feasible without an increase in surgically- or chemotherapyrelated complications. The median length of hospital stay was not statistically different in patients who received HIPEC and those who did not.

Time spent on the surgical procedure was slightly longer in the HIPEC group than in control one. This difference reached statistical significance. Even though other authors reported a $14 \%$ rate of postoperative complications after adjuvant HIPEC (11), in our experience, both surgical complications and chemotherapyrelated toxicity were low. None of the 14 patients showed peritoneal recurrence or distant metastases after a median follow-up of 12 months. Although the patients' sample size is too small to draw conclusions survival outcomes, these results are, however, in line with the encouraging results of other studies investigating the role of adjuvant HIPEC in high-risk CRC patients. These data support the need for a proper patient selection based on clinical criteria if surgeons plan to deliver HIPEC simultaneously with primary resection. These criteria include data from pre-operative imaging, histological biopsies, and intra-operative findings. However, in our experience, it was difficult to select patients based on clinical staging adequately. A well-defined cT4 stage based on imaging or intraoperative findings frequently turns out to have a pathological T3 stage. Therefore, this proactive management of patients with stage T4 as the only risk factor could represent an overtreatment recently suggested (30). Three trials, the Italian PROMENADE, The Chinese APEC, and the Spanish HIPECT4, are currently investigating the early use of adjuvant HIPEC for locally advanced CRC (3133). These studies are currently recruiting patients, with the latter using mitomycin-C, and hopefully shed more light on this matter.

\section{Conclusions.}

Colorectal surgery plus HIPEC treatment is safe and feasible in colorectal cancer patients at high-risk for peritoneal invasion, although clinically and radiologically undetectable. Our data concerning the impact of such a procedure on survival parameters are not available due to follow-up shortness. However, further studies are needed to better identify early peritoneal invasion and optimize the role of colorectal surgery plus HIPEC in patients at risk of peritoneal cancer spread.

\section{Author Contributions}

Conceptualization, Ina Macaione, Pietro Mezzatesta and Vittorio Gebbia; Data curation, Ina Macaione, Lucio Mandalà and Vittorio Gebbia; Formal analysis, Dario Piazza; Investigation, Ina Macaione, Lucio Mandalà, Antonella Campisi, Elena Roz, Sebastiano Mercadante and Pietro Mezzatesta; Methodology, Dario Piazza and Vittorio Gebbia; Writing - original draft, Vittorio Gebbia; Writing - review \& editing, Ina Macaione, Lucio Mandalà and Vittorio Gebbia.

Funding: This study was supported by La Maddalena Hospital.

Acknowledgments: The authors would like to thank all patients voluntarily taking part in the study.

Conflicts of Interest: V.G. received honoraria from Lilly, Roche, Janssen, GSK, and Pfizer for consultations and lectures unrelated to this project. D.P. has received honoraria from Astra Zeneca for consultations unrelated to this project.

\section{References.}

1. Bray F, Ferlay J, Soerjomataram I, et al. Global cancer statistics 2018: GLOBOCAN estimates of incidence and mortality worldwide for 36 cancers in 185 countries. CA. Cancer. J. Clin. 2018; 68: 394424.

2. Galandiuk S, Wieand HS, Moertel CG, et al. Patterns of recurrence after curative resection of carcinoma of the colon and rectum. Surg. Gynecol. Obstet. 1992; 174:27-32. 
3. Elferink MAG, de Jong KP, Klaase JM, et al. Metachronous metastases from colorectal cancer: a population-based study in north-East Netherlands. Int. J. Color. Dis. 2015; 30:205-12.

4. Franko J, Shi Q, Goldman CD, et al. treatment of colorectal peritoneal carcinomatosis with systemic chemotherapy: a pooled analysis of north central cancer treatment group phase III trials N9741 and N9841. J. Clin. Oncol. 2012; 30:263-7.

5. Hugen N, vandeVelde C.J., deWilt JH, Nagtegaal ID. Metastatic pattern in colorectal cancer is strongly influenced by histological subtype. Ann. Oncol. 2014; 25(3):651-657.

6. Segelman J, Granath F, Holm T, et al. Incidence, prevalence and risk factors for peritoneal carcinomatosis from colorectal cancer. Br. J. Surg. 2012; 99: 699-705.

7. van Gestel YRBM, Thomassen I, Lemmens VEPP, et al. Metachronous peritoneal carcinomatosis after curative treatment of colorectal cancer. Eur. J. Surg. Oncol. 2014; 40: 963-69.

8. Klaver CEL, van Huijgevoort NCM, de Buck van Overstraeten A, et al. Locally advanced colorectal cancer: true peritoneal tumor penetration is associated with peritoneal metastases. Ann. Surg. Oncol. 2018; 25: 212-20.

9. Cheynel N, Cortet M, Lepage C, et al. Incidence, patterns of failure, and prognosis of perforated colorectal cancers in a well-defined population. Dis. Colon. Rectum 2009; 52: 406-11.

10. Sugarbaker P.H. Peritonectomy procedures. Ann. Surg. 1995; 221:29-42.

11. Klaver CEL, Wisselink DD, Punt CJA, et al COLOPEC collaborators group. Adjuvant hyperthermic intraperitoneal chemotherapy in patients with locally advanced colon cancer (COLOPEC): a multicentre, open-label, randomised trial. Lancet Gastroenterol. Hepatol. 2019;4(10):761-770.

12. Elias D, Honoré C, Dumont F, et al. Results of systematic second look surgery plus HIPEC in asymptomatic patients presenting a high risk of developing colorectal peritoneal carcinomatosis. Ann. Surg. 2011;254:289-93.

13. Sloothaak DAM, Mirck B, Punt CJ, et al. Intraperitoneal chemotherapy as adjuvant treatment to prevent peritoneal carcinomatosis of colorectal cancer origin: a systematic review. Br. J. Cancer 2014;111(6):1112-1121.

14. Noura S, Ohue M, Shingai T. Effects of intraperitoneal chemotherapy with mitomycin c on the prevention of peritoneal recurrence in colorectal cancer patients with positive peritoneal lavage cytology findings. Ann. Surg. Oncol. 2011;18:396-404.

15. Sammartino P, Sibio S, Biacchi D, et al. Long-term results after proactive management for locoregional control in patients with colonic cancer at high risk of peritoneal metastases. Int. J. Color. Dis. 2014;29(9):1081-9.

16. Dindo D, Demartines N, Clavien PA. Classification of surgical complications: A new proposal with evaluation in a cohort of 6336 patients and results of a survey. Ann. Surg. 2004, 240: 205-213.

17. https://ctep.cancer.gov/protocolDevelopment/electronic applications/ctc.htm

18. Rosner B. Fundamentals of Biostatistics. 7th ed. Boston, MA: Brooks/Cole; 2011.

19. Ivanova, A., Qaqish, B.F., and Schell, M.J. Continuous toxicity monitoring in phase II trials in oncology. Biometrics 2005, 61: 540-545.

20. Faron M, Macovei R, Goéré D, Honoré C, et al. Linear relationship of peritoneal cancer index and survival in patients with peritoneal metastases from colorectal cancer. Ann. Surg. Oncol. 2016; 23: 114-19.

21. Sugarbaker P.H. Management of peritoneal metastases - Basic concepts. J. BUON. 2015, 20 Suppl 1:S2-11.

22. Mangan C, Moinuddin Z, Summers A, de Reuver P, van Dellen D, Augustine T. Encapsulating peritoneal sclerosis following hyperthermic intraperitoneal chemotherapy. ANZ. J. Surg. 2018; published online Sept 2.

23. Dohan A, Hoeffel C, Soyer P, et al. Evaluation of the peritoneal carcinomatosis index with C.T. and MRI. Br. J. Surg. 2017; 104: 1244-49.

24. Sugarbaker PH, Stuart OA. Perioperative FOLFOX in management of peritoneal metastases of colorectal cancer. Case report of 2 patients. Int. J. Surg. Case. Rep. 2020;75:279-285.

25. André T, Boni C, Navarro M, Tabernero J, Hickish T, Topham C, Bonetti A, Clingan P, Bridgewater J, Rivera F, de Gramont A. Improved overall survival with oxaliplatin, fluorouracil, and leucovorin 
as adjuvant treatment in stage II or III colon cancer in the MOSAIC trial. J. Clin. Oncol. 2009, 27(19):3109-16.

26. Colucci G, Gebbia V, Paoletti G, Giuliani F, Caruso M, Gebbia N, Cartenì G, Agostara B, Pezzella G, Manzione L, Borsellino N, et al; Gruppo Oncologico Dell'Italia Meridionale. Phase III randomized trial of FOLFIRI versus FOLFOX4 in the treatment of advanced colorectal cancer: a multicenter study of the Gruppo Oncologico Dell'Italia Meridionale. J. Clin. Oncol. 2005, 23(22):4866-75.

27. Tan G, Wong J. Surgical management and hyperthermic intraperitoneal chemotherapy for locally advanced colorectal cancer. J. Gastrointest. Oncol. 2020, 11(3):508-512.

28. Klaver CEL, Stam R, Sloothaak DAM, Crezee J, Bemelman WA, Punt CJA, Tanis PJ. Colorectal cancer at high risk of peritoneal metastases: long term outcomes of a pilot study on adjuvant laparoscopic HIPEC and future perspectives. Oncotarget. 2017, 8(31): 51200-51209.

29. Goéré D, Glehen O, Quenet F, Guilloit JM, Bereder JM, Lorimier G, Thibaudeau E, Ghouti L, Pinto A, Tuech JJ, et al; BIG-RENAPE group. Second-look surgery plus hyperthermic intraperitoneal chemotherapy versus surveillance in patients at high risk of developing colorectal peritoneal metastases (PROPHYLOCHIP-PRODIGE 15): a randomised, phase 3 study. Lancet Oncol. 2020, 21(9):1147-1154.

30. Arrizabalaga NB, Navascués JME, Echaniz GE, Ansorena YS, Galán CP, Martín XA, Pardo LV. Prophylactic HIPEC in pT4 Colon Tumors: Proactive Approach or Overtreatment? Ann. Surg. Oncol. 2020, 27(4):1094-1100.

31. Sammartino P, Biacchi D, Cornali T, Cardi M, Accarpio F, Impagnatiello A, Sollazzo BM, Di Giorgio A. Proactive Management for Gastric, Colorectal and Appendiceal Malignancies: Preventing Peritoneal Metastases with Hyperthermic Intraperitoneal Chemotherapy (HIPEC). Indian J. Surg. Oncol. 2016, 7(2):215-24.

32. Adjuvant Hyperthermic Intraperitoneal Chemotherapy (HIPEC Versus no HIPEC in Locally Advanced Colorectal Cancer (APEC Study). www. clinicaltrials.gov > NCT02965248

33. Arjona-Sánchez A, Barrios P, Boldo-Roda E, et al. HIPECT4: multicentre, randomized clinical trial to evaluate safety and efficacy of Hyperthermic intra-peritoneal chemotherapy (HIPEC) with Mitomycin C used during surgery for treatment of locally advanced colorectal carcinoma. BMC Cancer. 2018, 18(1):183. 